\title{
A Conversation Model Enabling Intelligent Agents to Give Emotional Support
}

\author{
Janneke M. van der Zwaan, Virginia Dignum, and Catholijn M. Jonker \\ Delft University of Technology, The Netherlands \\ $\{j \cdot m$.vanderzwaan, m.v.dignum, c.m.jonker\}@tudelft.nl
}

\begin{abstract}
In everyday life, people frequently talk to others to help them deal with negative emotions. To some extent, everybody is capable of comforting other people, but so far conversational agents are unable to deal with this type of situation. To provide intelligent agents with the capability to give emotional support, we propose a domain-independent conversational model that is based on topics suggested by cognitive appraisal theories of emotion and the 5-phase model that is used to structure online counseling conversations. The model is implemented in an embodied conversational agent called Robin.
\end{abstract}

\section{Introduction}

To alleviate stress and deal with negative emotions, people frequently turn to others to talk about their problems. Most people are more or less successful in comforting others. Early work in the field of affective computing demonstrated that virtual agents are able to reduce negative emotions in users [5]. More recent developments show that empathic agents are increasingly capable of complex social and emotional dialogues, but so far they are unable to comfort users.

We are interested in investigating how and to what extent conversational agents can provide social support. Social support or comforting refers to communicative attempts to alleviate the emotional distress of another person [3]. Our research concerns the design and evaluation of an Embodied Conversational Agent (ECA) that provides social support to victims of cyberbullying [11] ${ }^{1}$. Cyberbullying is bullying through electronic communication devices [6]. Although in our application we assume the user's problem is connected to cyberbullying, we want to keep the strategies for giving social support as domain-independent as possible.

General advice on comforting includes letting the other party talk about their 'thoughts and feelings' regarding the upsetting situation [10]. However, this does not specify what topics might be addressed. To overcome this gap, Burleson and Goldsmith linked comforting to cognitive appraisal theories of emotions and identified a sequence of topics in comforting conversations [3]. Our agent uses

\footnotetext{
1 This work is funded by NWO under the Responsible Innovation program via the project 'Empowering and Protecting Children and Adolescents Against Cyberbullying'.
} 
this model to determine what topic it will bring up next. Because the model is based on a theory of how emotions arise in humans, it allows the agent be aware of emotional processes in humans and to adjust its actions accordingly. The goal of this paper is to present a domain-independent conversational model of comforting. We also introduce our implementation of this model in a conversational agent called Robin.

The agent and the user communicate predominantly through natural language text messages. Given the complexity of interpreting and generating natural language, in the current system, the text interpretation and generation have not been implemented. Instead, we use logical representations of utterances (speech acts). This abstraction allows us to focus on the agent's reasoning process and show how it can use appraisal theory to give social support.

This paper is organized as follows. In the next section, we provide a background on emotion theories. In Sect. 3, we present the conversation model. Section 4 introduces the comforting agent that implements the conversation model. Section 5 presents our conclusions.

\section{Background}

To assist users in dealing with their negative emotions, the agent needs be aware of the user's emotional state and the reasons these emotions arise. Within the field of psychology different emotional theories exist. Two major strands of emotion theories that can be distinguished are dimensional theories [4] and cognitive appraisal theories of emotion [8].

Dimensional emotion theories are based on the idea of classifying emotions along an arbitrary amount of dimensions of connotative meaning. A well known dimensional model is the Pleasure, Arousal, Dominance (PAD) model of emotions, which assumes an emotion (more precisely: affect) can be defined as a coincidence of values on the dimensions pleasure, arousal and dominance $[7,9]$. Pleasure (valence) determines how positive or negative the emotion, mood or attitude is. Arousal describes whether the emotion, mood or attitude involves activation or deactivation. Dominance refers to the degree to which the individual feels in control versus feels submissive with regards to the stimulus or situation. For example, anger would be low pleasure, high arousal, high dominance, while sadness would be low pleasure, low arousal, low dominance.

The basic assumption of cognitive appraisal theories is that emotions are triggered by the evaluation of a stimulus with respect to several criteria, including personality dimensions, characteristics of the situation, and other context variables [8]. The evaluation of a stimulus is called appraisal. Different cognitive theories of appraisal exist, they differ mostly in the dimensions that make up the appraisal process. Ortony, Clore and Collins proposed a computationally friendly formalization of cognitive appraisal theories [8]. The so-called OCC model is frequently used as a model for computerized emotion.

Burleson and Goldsmith use Lazarus' cognitive appraisal theory of emotions to explain how the process of comforting works [3]. After observing emotions are 
elicited by appraisals of situations and not by the situations themselves, they conclude "the only way a distressed emotional state can be altered is through an individual changing the appraisals that underlie and constitute the emotional distress" [3]. Helpers can facilitate these reappraisals by encouraging the distressed person to explore and clarify his thoughts and feelings that are relevant to the stressful situation. As a result, the distressed person may change his goals, views of the situation, and/or coping efforts [3].

Based on these observations, Burleson and Goldsmith suggest a set of topics that can be addressed in the course of a supportive conversation. First, the conversation should focus on getting a fuller appreciation of the emotional state, the reasons for its occurrence, and an assessment of its appropriateness. After that, the current coping strategy and its effectiveness can be discussed. Finally, if the current coping strategy does not seem to solve the problem, alternative coping strategies can be explored [3].

To further structure the conversation between the agent and the user, the conversational topics identified by Burleson and Goldsmith will be linked to phases in the 5-phase model. The 5-phase model was developed as a methodology to structure counseling conversations via telephone and chat [1]. The five phases of a conversation are: 1) Warm welcome: the counselor connects with the child and invites him to explain what he wants to talk about; 2) Gather information: the counselor asks questions to try to establish the problem of the child; 3) Determine the objective of the session: the counselor and the child determine the goal of the conversation (e.g., getting tips on how to deal with bullying); 4) Work out the objective: the counselor stimulates the child to come up with a solution; and 5) Round off: the counselor actively rounds off the conversation.

\section{Conversation Model}

To get a model for comforting conversations, we combined the structure of the 5 -phase model with the topics Burleson and Goldsmith suggested based on appraisal theory. Table 1 shows the division of the topics among the phases. In the explanation below, the term comforter refers to the agent and the distressed person is the user.

In the first conversation phase (welcome) the comforter greets the distressed person. In phase 2 (gather information), the comforter first asks the distressed person questions to identify facts about the event that elicits negative emotions. The second topic is the distresses person's emotional state. To be able to determine whether the distressed person needs to cope, the comforter also registers the intensity of the emotion. If the emotional state is negative, appraisal theory suggests a personal goal is threatened. Subsequently, the comforter asks the distressed person to specify which goal is threatened. After that, the comforter asks more details about the event. These details (together with information already acquired) determine the advice the comforter can give in phase 4 . Which details are relevant and how details are mapped to advice depends on the domain in which the agent operates. When the comforter has gathered sufficient infor- 


\begin{tabular}{|l|l|}
\hline Conversation phase & Topic(s) \\
\hline 1) Welcome & Hello \\
\hline 2) Gather information & Event (general) \\
& Emotional state \\
& Personal goal \\
& Event (details) \\
& Coping (current) (if need to cope) \\
\hline 3) Determine conversation objective & Conversation objective \\
\hline 4) Work out objective & Coping (future) (if need to cope) \\
& Advice (depending on conversation objective) \\
\hline 5) Round off & Bye \\
\hline
\end{tabular}

Table 1. Conversation phases and topics.

mation (what is sufficient also depends on domain knowledge) and believes the distressed person needs to cope, it moves on to discussing the distressed person's current coping strategy. Then phase 3 (determine conversation objective) starts and the comforter asks the distressed person what he or she wants to accomplish with the conversation. Multiple objectives are possible; for simplicity we assume the distressed person either wants to tell his story or get advice to deal with the problem. For both conversation objectives, phase 4 (work out objective) starts with discussing the distressed person's future coping options. If the distressed person wants advice on how to deal with his problem, subsequently the comforter gives advice. Finally, in phase 5 (round off), the comforters says goodbye to the distressed person.

\section{Robin, the Comforting Agent}

The conversation model introduced in the previous section was implemented in an embodied conversational agent called Robin. Robin tries to empower victims of cyberbullying by giving emotional support and practical advice. Figure 1 shows a screenshot of the system. The user communicates with Robin through a chat interface. As mentioned before, in the current implementation, verbal communication consists of speech acts instead of natural language utterances. The agent's embodiment consists of a set of pictures of the iCat (top left of Fig. 1). To communicate his emotional state, the user manipulates the AffectButton [2] (bottom left of Fig. 1). The AffectButton is a button with a rudimentary and gender-neutral face that changes its expression based on the position of the mouse cursor. By clicking the button when it shows the emotional expression the user wants to communicate, the corresponding PAD values are send to the agent. The emotion input is further explained in Sect. 4.1.

The input of the agent consists of speech acts and PAD values. The output consists of speech acts and one of five discrete emotional expressions (happy, surprised, sad, angry, afraid). 


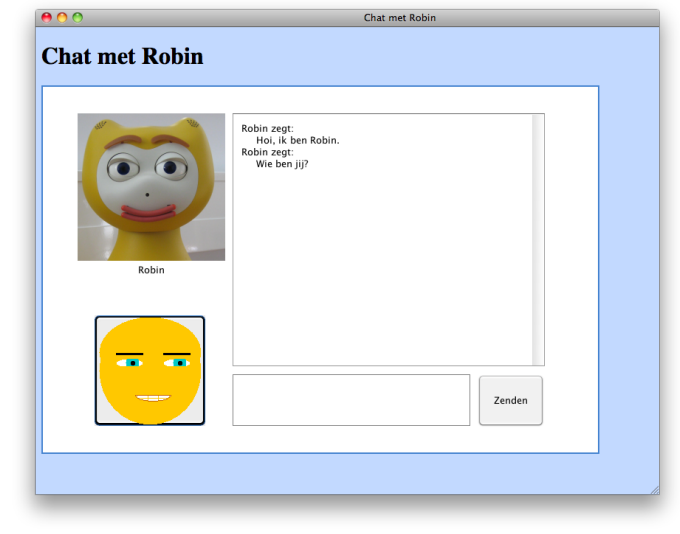

Fig. 1. Screenshot of Robin, the comforting agent.

\subsection{Emotion Input}

To gather information about the user's current emotional state, Robin uses the AffectButton (see Fig. 1). The AffectButton is a tool for explicit affective feedback. Since the user's emotional state is explicitly being addressed during the conversation, explicit emotion input arguably is more suitable than implicit emotion input (e.g., by recognizing facial expressions), because it requires the user to consciously think about his emotions. The AffectButton sends PAD triplets to the agent's reasoning engine.

To be able to interpret emotional states, PAD triplets have to be mapped to OCC emotion types. Since PAD and OCC are based on two different strands of emotion theories, no fixed mapping between these two models exists. Therefore, we propose a generic mapping. Based on the assumption that the user is dealing with a negative event all input emotions are mapped to well-being emotions. The value for pleasure determines whether an emotion is positive or negative; positive emotions $(\mathrm{P} \geq 0)$ are mapped to the emotion type joy and negative emotions $(\mathrm{P}<0)$ are mapped to distress. The value of $\mathrm{P}$ also determines the intensity of the emotion: $-1 \leq \mathrm{P}<-0.66$ or $0.66<\mathrm{P} \leq 1$, high intensity; $-0.66 \leq \mathrm{P}$ $<-0.33$ or $0.33<\mathrm{P} \leq 0.66$, medium intensity; $-0.33 \leq \mathrm{P}<0$ or $0 \leq \mathrm{P}$ $\leq 0.33$, low intensity. The agent assumes coping is necessary for medium and high negative emotions.

\subsection{Emotion Output}

Emotion output is realized by the embodiment and consists of one of five discrete emotional expressions (happy, surprised, sad, angry, afraid). The default expression for the agent is happy. Each time the user communicates an emotional expression with the AffectButton, the agent verbally confirms and mirrors this emotional state. After confirming the user's emotional state, the facial expression changes back to its default expression (happy). 


\section{Conclusion}

In this paper, we presented a domain-independent dialogue model for comforting conversations. The model combines the 5-phase model, used to structure counseling conversations, with topics that are relevant according to cognitive appraisal theories of emotion. We also introduced our implementation of this model in a conversational agent that provides social support to victims of cyberbullying.

The agent uses the AffectButton to obtain information about the user's emotional state. The AffectButton is based on a dimensional emotion model, whereas the conversational model is based on appraisal theories. Another contribution of this paper is a mapping from PAD values to OCC emotion types.

The conversation model does not yet lead to complete conversations. To make the comforting conversations proceed smoothly, additional conversation

techniques are required, such as requesting feedback, summarizing, verbalizing feelings, giving compliments, encouraging the conversation partner and (sympathetically) acknowledging what he or she says. For future work, we plan to equip the agent with these essential conversation skills.

\section{References}

1. A. de Beyn. In gesprek met kinderen:de methodiek van de kindertelefoon. SWP, 2003.

2. J. Broekens and W.P. Brinkman. Affectbutton: Towards a standard for dynamic affective user feedback. In Affective Computing and Intelligent Interaction (ACII) 2009, 2009.

3. B.R. Burleson and D.J. Goldsmith. Handbook of Communication and Emotion: Research, Theory, Applications, and Contexts, chapter How the Comforting Process Works: Alleviating Emotional Distress through Conversationally Induced Reappraisals, pages 245-280. San Diego, CA, US: Academic Press, 1998.

4. T.L. Gehm and K.R. Scherer. Facets of emotion: Recent research., chapter Factors determining the dimensions of subjective emotional space, pages 99-113. Hillsdale, NJ, England: Lawrence Erlbaum Associates, Inc, 1988.

5. K. Hone. Empathic agents to reduce user frustration: The effects of varying agent characteristics. Interact. Comput., 18(2):227-245, 2006.

6. Q. Li. New bottle but old wine: A research of cyberbullying in schools. Computers in Human Behavior, 23(4):1777-1791, 2007.

7. A. Mehrabian. Basic Dimensions for a General Psychological Theory. Oelgeschlager, Gunn \& Hain, Cambridge, 1980.

8. A. Ortony, G.L. Clore, and A. Collins. The cognitive structure of emotions. Cambridge Univ. Press, 1988.

9. C.E. Osgood. Dimensionality of the semantic space for communication via facial expressions. Scandinavian Journal of Psychology, 7(1):1-30, 1966.

10. J.W. Pennebaker. Putting stress into words: Health, linguistic, and therapeutic implications. Behaviour Research and Therapy, 31(6):539-548, 1993.

11. J.M. van der Zwaan, V. Dignum, and C.M. Jonker. Simulating peer support for victims of cyberbullying. In Proceedings of the 22st Benelux Conference on Artificial Intelligence (BNAIC 2010), 2010. 